\title{
Performance Characteristics of the lonospheric Neutron Content Analyzer (INCA)
}

\author{
J. G. Mitchell*1,2, A. Bruno ${ }^{1}$, Candey, $\mathbf{S}^{1}$, G.A. de Nolfo ${ }^{1}$, J. Dumonthier ${ }^{1}$, I. \\ Liceaga-Indart $^{1,3}$, I. Rankin ${ }^{4}$, K. Rankin ${ }^{5}$, J.M. Ryan ${ }^{6}$, S. Stochaj ${ }^{4}$, G. Suarez ${ }^{1}$ \\ ${ }^{1}$ NASA Goddard Space Flight Center, Greenbelt, MD \\ E-mail: john.g.mitchellenasa.gov \\ ${ }^{2}$ The George Washington University, Department of Physics, Washington D.C. \\ ${ }^{3}$ The Catholic University of America, Washington D.C. \\ ${ }^{4}$ Klipsch School of Electrical and Computer Engineering, New Mexico State University, Las \\ Cruces, NM \\ ${ }^{5}$ Department of Mechanical and Aerospace Engineering, New Mexico State University, Las \\ Cruces, NM \\ ${ }^{6}$ Space Science Center, University of New Hampshire, Durham, NH
}

\begin{abstract}
The Ionospheric Neutron Content Analyzer (INCA) is a double scatter neutron spectrometer designed for a $3 \mathrm{U}$ CubeSat, currently set for an Educational Launch of Nanosatellites (ELaNa) launch in 2019. In a double scatter neutron spectrometer, a neutron scatters in two separate scintillators (D1, D2) spaced $15 \mathrm{~cm}$ apart, giving information on the incident neutron's direction and energy from the pulse height in D1 and time-of-flight between the scintillators. INCA employs silicon photomultiplier (SiPM) arrays coupled to p-terphenyl scintillators. A neutron scattering event in p-terphenyl results in a pulse shape with a longer tail, due to delayed fluorescence of the scintillator, than that of a gamma ray. This property, known as pulse shape discrimination, is utilized in INCA to distinguish detected neutrons from gamma rays. Charged particles are vetoed by plastic scintillator anticoincidence detectors completely surrounding the spectrometer. The instrument was calibrated using a ${ }^{252} \mathrm{Cf}$ neutron beam, and compared to Geant 4 simulation results. Details of the INCA instrument and these calibrations are discussed.
\end{abstract}

36th International Cosmic Ray Conference -ICRC2019-

July 24th - August 1st, 2019

Madison, WI, U.S.A.

${ }^{*}$ Speaker. 


\section{Scientific Motivation}

The Van Allen radiation belts are concentrations of energetic charged particles trapped by the Earth's magnetic field. The inner radiation belt, from some hundreds to some thousands of $\mathrm{km}$ above the Earth's surface, consists primarily of protons with energies up to a few $\mathrm{GeV}$, with a small component of electrons, predominantly of energies less than $1 \mathrm{MeV}$. A full understanding of the source and composition of the protons trapped in the Van Allen belts is of practical, as well as scientific, importance as particle radiation poses risks to both astronauts and spacecraft equipment [4]. The main source of the proton population of the inner radiation belt is the decay of upward-moving, albedo, neutrons [12] produced by interactions of interplanetary cosmic rays with the Earth's atmosphere [1][7]. These albedo neutrons undergo beta decay, from which protons are produced. This is the basis of the cosmic ray albedo neutron decay (CRAND) theory [5][10] that albedo neutrons populate the inner radiation belt with high-energy protons, as well as high energy electrons [11].

Neutrons detected at the Earth can also be produced at the Sun. Solar neutrons are particularly interesting as they are by-products of interactions of accelerated ions with the dense solar atmosphere within flares. Neutrons are produced by the process of spallation, in which an accelerated proton or ion interacts with the nucleus of an atom, ejecting nucleons by inelastic scattering, and leaving an unstable residual nucleus that must decay by emission of more nucleons or clusters of nucleons. A variety of particles may emerge from the interaction, including neutrons, at velocities that can exceed that of the incident particle due to the effect of Fermi motion in the target nucleus. A host of secondary particles are released as a result of these interactions in addition to neutrons, including lighter nuclei and characteristic gamma-rays. Detecting these secondaries and characterizing their properties helps to constrain particle acceleration mechanisms taking place at the flare.

\section{Neutron Measurement Techniques}

The Ionospheric Neutron Content Analyzer (INCA) is a double scatter neutron spectrometer designed to detect and measure neutrons from the Sun and the Earth. It has been engineered to fit onto a $3 \mathrm{U}$ CubeSat bus. Double scatter neutron spectrometers employ two widely-spaced detectors to reconstruct the energy and determine the incident direction of a neutron by a combination of scattering direction, scattered proton energy, and time-of-flight measurements. In the double-scatter technique, a neutron scatters in two separate scintillators spaced relatively far apart (Figure 1). The detectors are typically comprised of organic scintillating material, either in plastic, crystalline or liquid form. In a scintillator, incident neutrons may elastically scatter off a hydrogen nucleus resulting in emission of a "recoil" proton carrying a considerable fraction of the incident neutron energy (up 
to the full neutron energy, depending on the scattering angle). The scintillators are viewed by sensitive photosensors, traditionally vacuum photomultiplier tubes (PMT), that detect the scintillation light and produce current pulses proportional to the detected light. These current pulses are fed to appropriate amplifiers and then digitized to measure both the amplitudes and arrival times of the light pulses. Information about the incident neutron's direction and energy is based on pulse height and time-of-flight. The incident energy of the neutron is the sum of the energy of the recoil proton in the first scintillator and the energy of the scattered neutron, measured by the neutron flight velocity. High-energy neutrons are challenging to measure, only using the energy of the recoil proton, because the recoil neutron easily escapes the detector, resulting in decreased accuracy in the energy measurement [6][8]. In addition, the elastic cross-section decreases with increasing neutron energies, so there are fewer double-scatter events at higher energies, although the recoil proton trajectories are longer, and so more detectable.

\section{Enabling Technologies}

PMTs have the disadvantage that they are typically large and require high bias voltages (several hundreds to a few thousand volts). As technology has moved toward the miniaturization of electronics, so too have photosensors. Silicon photomultipliers (SiPM) are solid-state photon detectors made up of an array of a large number of small micro-pixels, each on the order of tens of micrometers, that operate in limited Geiger mode. When a photon interacts in one of the micro-pixels, a current pulse is produced that reflects only the design and bias (tens of volts) of the micro-pixel. However, this current may contain $10^{6}$ electrons from detection of a single photon. The full output of the SiPM for a light pulse is the sum of the currents from all micro-pixels that detected a photon. Thus, the SiPM functions as a nearly proportional photo-detector with an effective gain on the order of $10^{6}$. SiPMs are much smaller, and require a far lower bias voltage than PMTs (tens of volts as opposed to thousands of volts). This makes them ideally suited for use in small spacecraft, especially CubeSats, that do not have room for PMTs or the power supplies required to bias PMTs. Double-scatter neutron detectors, such as INCA, can employ arrays of SiPMs coupled to the scintillators, enabling an entire double scatter neutron spectrometer to fit in a $3 \mathrm{U}$ CubeSat.

INCA utilizes two $5 \mathrm{~cm}$ cylindrical p-terphenyl scintillating crystals (D1 and D2) spaced $15 \mathrm{~cm}$ from center to center (see scintillator and SiPM array in Figure 2). P-terphenyl is a crystalline organic scintillator of density $1.23 \mathrm{~g} \mathrm{~cm}^{-3}$ and a $\mathrm{H} / \mathrm{C}$ ratio of 0.778. A neutron scattering event in p-terphenyl results in a pulse shape with a longer tail, due to delayed fluorescence of the scintillator, than that of a gamma ray. The number of photons emitted at a given time due to an interaction is given by

$$
N=A e^{-t / \tau_{f}}+B e^{-t / \tau_{s}}
$$

Where $\mathrm{A}$ and $\mathrm{B}$ are constants, $\tau_{f}$ corresponds to the fast decaying

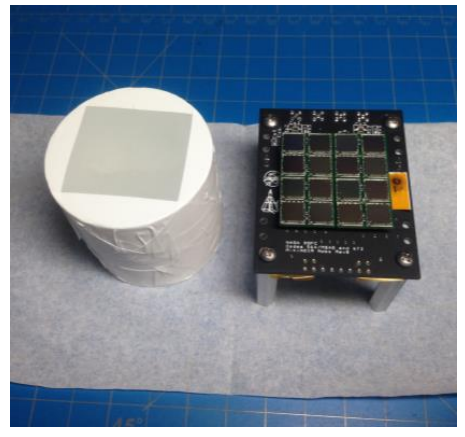

Figure 2: $5 \mathrm{~cm}$ p-terphenyl crystal with 4x4 SiPM array time, of order $1 \mathrm{~ns}$, and $\tau_{s}$ corresponds to the slow decaying time, up to $1 \mu \mathrm{s}$. This property, known as pulse shape discrimination (PSD), is utilized in INCA to distinguish detected neutrons from 
gamma rays. Both D1 and D2 are read out by $4 \mathrm{x} 4$ arrays of $6 \mathrm{~mm}$ square SiPMs by SensL. A timesampling application specific integrated circuit (ASIC), the DRS4 by Paul Scherrer Institut, was used for data readout to provide waveform capture, and thus enable PSD. The ratio of the integrated current pulse to its tail amplitude provides a measure of the pulse shape, enabling neutron/gammaray discrimination. The DRS4 also provides a measure of the time of flight (ToF) by computing the onset using digital constant fraction discrimination. D1 and D2 are surrounded by a $4 \pi \mathrm{sr}$ anti-coincidence detector (ACD) consisting of panels of $6 \mathrm{~mm}$ thick plastic scintillating panels, optically coupled to wavelength shifting bars. Wavelength shifting bars are read out at the beveled corners by $6 \mathrm{~mm}$ SiPMs (Figure 9).

\section{Performance Characteristics}

INCA was exposed to laboratory gamma-ray and neutron sources to calibrate and characterize the spectrometer and ACD. A pulse height to electron equivalent energy $\left(E_{e e}\right)$ conversion was determined using laboratory gamma-ray sources of known energy (Figure 3). These sources were also used to determine the relative energy resolution of a single p-terphenyl crystal using the empirically derived relation for a Compton edge

$$
\frac{\Delta E}{E}=1.5 \frac{C_{1 / 2}-C_{\max }}{C_{1 / 2}}
$$

as derived in Dietze \& Klein [2] (Figure 4). Here $C_{\max }$ is the position of the Gaussian peak, and $C_{1 / 2}$ is the half-maximum position on the falling edge. It is noted that according to simulations performed using Geant 4 (discussed below), the gamma-ray energy at which photoelectric absorption and Compton scattering have equal influence is $\sim 22 \mathrm{keV}$. At that transition point, the energy resolution degrades significantly due to this effect, and the increased influence of electronic noise. As outlined in Sardet et al. [9], energy resolution data were fit using

$$
\frac{\Delta E}{E}=\sqrt{\alpha^{2}+\frac{\beta^{2}}{E}+\left(\frac{\gamma}{E}\right)^{2}}
$$

where $\alpha$ corresponds to the transmission of light from the scintillator to the SiPM, $\beta$ corresponds to statistical effects throughout the system, and $\gamma$ incorporates noise effecting energy resolution. The optimal fit to our data was found for parameters $\alpha=12.91, \beta=4.14 \times 10^{-3}, \gamma=16.18$.

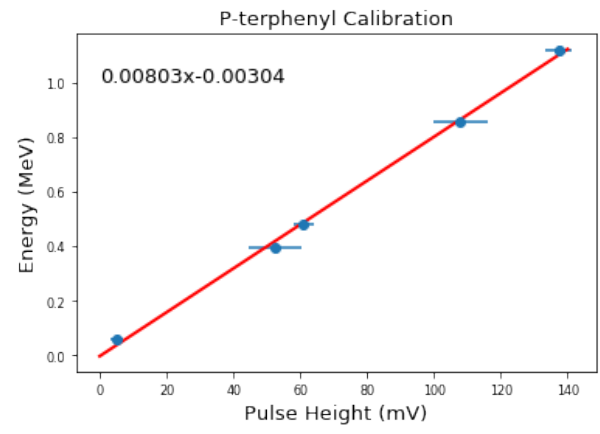

Figure 3: Peak voltage to $E_{e e}$ calibration from a variety of laboratory gamma-ray sources of known energy.

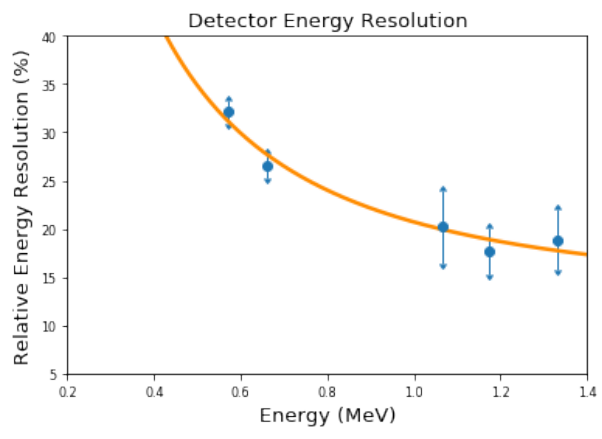

Figure 4: Relative energy resolution of p-terphenyl. In general, energy resolution improves with increasing gamma-ray energy. 
The instrument was calibrated using a tightly collimated ${ }^{252} \mathrm{Cf}$ neutron beam. A response function for proton interactions in p-terphenyl was created using the time-of-flight of neutrons between the two detectors. A sharp drop-off would be expected in an ideal detector, corresponding to the maximum energy that can be imparted to the recoil proton, given the incident neutron energy from the ToF. These preliminary results are consistent with Sardet et al. [9], although the statistics are poor and it is likely that multiple neutrons entered the coincidence window given the proximity and high activity of the source (Figure 5). Future plans include more thorough calibration using the INCA spare.

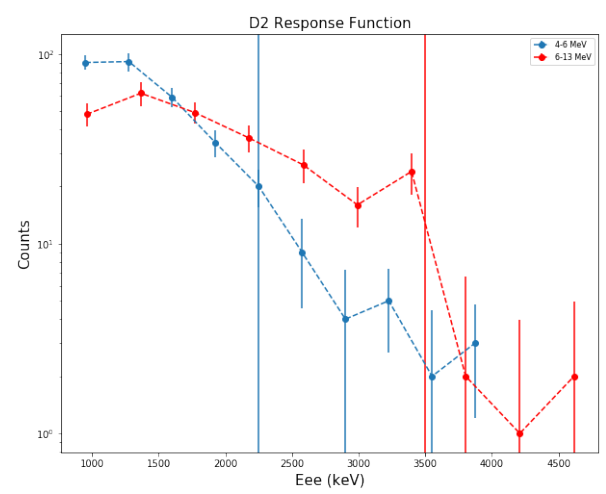

Figure 5: Neutron response function created by binning events based on ToF. Drop-off point for a given energy from [9] shown as vertical lines.

As described above, PSD is employed to distinguish between neutrons and gamma-rays. The use of p-terphenyl has been effective for these purposes. The time-sampling DRS4 ASIC by Paul Scherrer Institut allows fine time resolution enabling accurate measurements of neutron time of flight (ToF) between D1 and D2. Neutrons exhibit a significantly longer ToF than gamma-rays. INCA established a time resolution of $\sim 620$ ps for events with $\mathrm{MeV}_{e e}$ greater than 1.6 (Figure 6).
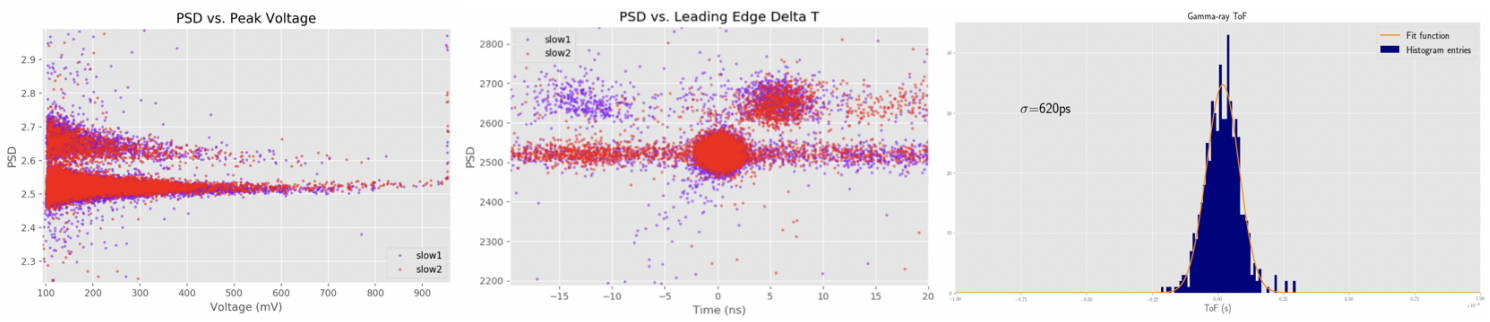

Figure 6: Left: PSD vs peak height in p-terphenyl scintillator. Slow1/2 refers to slow output of D1 and D2 respectively. Group of points with higher PSD correspond to neutrons. Middle: PSD vs. ToF in p-terphenyl scintillator. Slow 1/2 refers to slow output of D1 and D2 respectively. Neutrons are characterized by longer ToF and higher PSD value. Right: ToF between D1 and D2 of gamma-rays. Time resolution is $\sim 620$ ps for events with $\mathrm{MeV}_{e e}$ greater than 1.6

Incident ${ }^{252} \mathrm{Cf}$ neutron kinetic energy was reconstructed as described above and fit to a Watt spectrum

$$
N(E)=A e^{-b E} \sinh (\sqrt{c E})
$$

with parameters $A=2500, b=0.88$, and $c=2$ (Figure 7), in agreement with Smith et al. [13]. Due to the high trigger threshold, the Watt spectrum fit was applied only to the falling edge. Future plans include a calibration run at a lower trigger threshold using the INCA spare. 


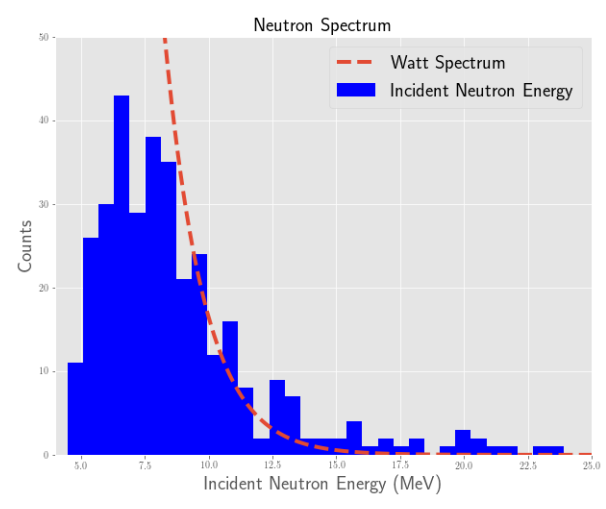

Figure 7: Measured ${ }^{252} \mathrm{Cf}$ spectrum

Charged particles are distinguished from neutral particles by the aforementioned ACD. Due to the thinness of the ACD, neutral particles predominantly pass through the ACD panels without interacting in the material, while charged particles consistently interact causing scintillation. In this way, a hit in both the ACD and the spectrometer detectors can be "vetoed" as a charged particle during analysis. The veto will be an important component in discriminating against the high proton and electron environment of low Earth orbit (LEO). The spatial detection efficiency of the ACD has been measured using laboratory radiation sources, with an overall variation between the center and corners of a given panel of less than $16 \%$ (Figure 8 ).

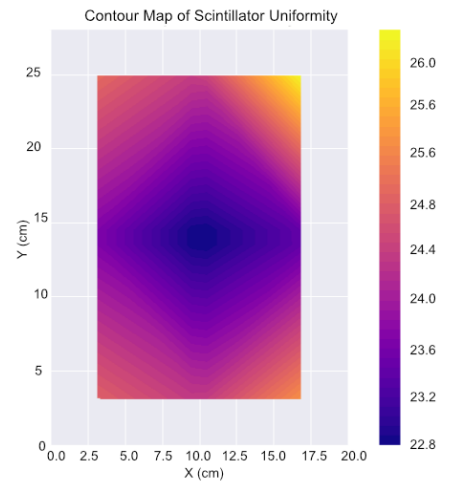

Figure 8: "Heat" map of average peak height of ACD panel. Toward each corner, peak pulse height increases due to proximity of the interaction with SiPMs located at corner. Courtesy of summer intern: Scott Candey

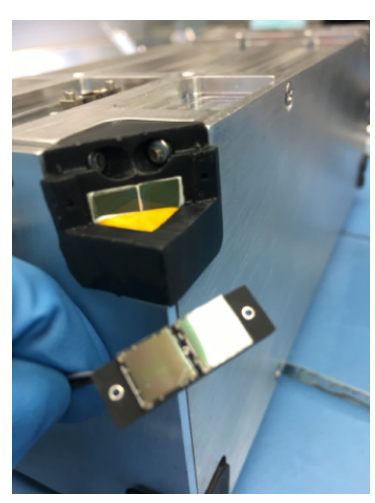

Figure 9: Full INCA spectrometer/ACD in spacecraft housing. 1x2 SiPM array removed to expose beveled corner of wavelength shifting bars.

\section{Simulation}

MEGAlib, a suite of Geant4 simulation tools [14], has been employed to simulate INCA. Simulations of the response of representative p-terphenyl crystals to a variety of laboratory sources was compared with actual data to optimize the simulation (Figure 10). 

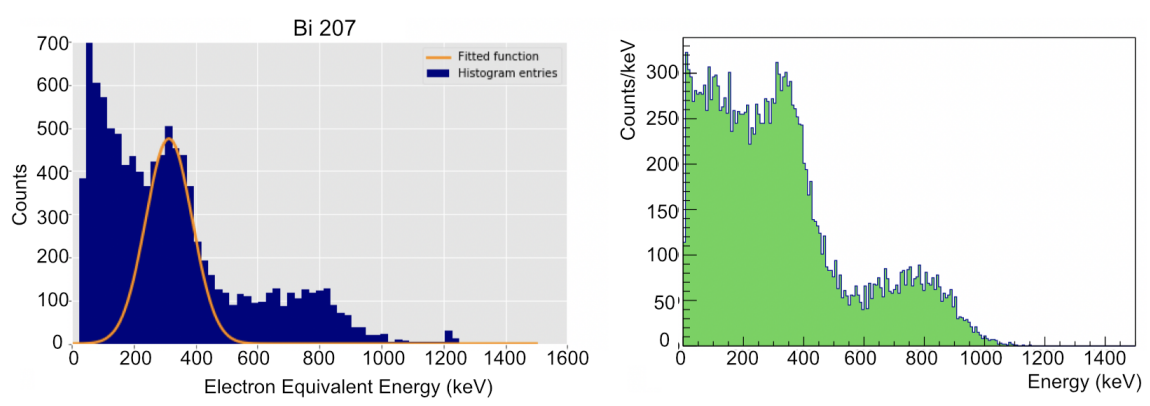

Figure 10: Example of agreement between data (left) and simulation (right) of the detected spectrum of a laboratory ${ }^{207} \mathrm{Bi}$ gamma-ray source

Following successful simulation of a single p-terphenyl crystal, the simulation was expanded to include the full neutron spectrometer along with the ACD (Figure 11). Analysis of simulation results and comparison to ${ }^{252} \mathrm{Cf}$ calibration run are ongoing.

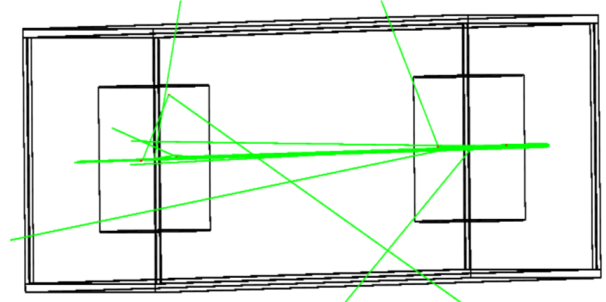

Figure 11: Simulation of INCA in mono-energetic neutron beam. Incident neutrons are injected from right side and may interact in D1 (right) before scattering to D2 (left).

\section{INCA Mission}

The INCA CubeSat mission is a $3 \mathrm{U}$ CubeSat built as a collaboration between New Mexico State University (NMSU), and NASA Goddard Space Flight Center (NASA/GSFC). INCA has been manifested for launch in 2019 on NASA's Educational Launch of Nanosatellites (ELaNa) 20 mission, through the CubeSat Launch Initiative (CSLI) on a Virgin Orbit LauncherOne rocket. The INCA satellite will be deployed from a Xtenti $3 \mathrm{U}$ canister, capable of deploying CubeSats built to the CalPoly CubeSat standard. The INCA mission is first a technology demonstration and second a science mission, aimed at better understanding the radiation environment in LEO using modern scintillators and a SiPM-based readout to construct a double scatter neutron spectrometer. The goal is to directly measure neutrons in the Earth's atmosphere and solar neutrons. In the interest of achieving this science, INCA will first demonstrate the functionality of its scintillator and SiPM-based neutron detector. The SiPM's on this detector have not been flown in space, and an important value of INCA will be to raise their technical readiness level (TRL). Once the neutron detector has been proven functional, INCA will use the detector to measure the neutron spectrum in LEO, correlating the data with measurement time and latitude. INCA will fly in a highly inclined orbit for a period of several months, in the hopes of witnessing an energetic solar event, and to correlate it with changes in the neutron local environment. 
The INCA CubeSat has a mass of only $4.08 \mathrm{~kg}$, and employs 4 deployable solar arrays and 4 side panel arrays, for a combined area of over $17,300 \mathrm{~cm}^{2}$, to generate on average $11 \mathrm{~W}$ of power per orbit. The spectrometer is expected to consume $9.5 \mathrm{~W}$ throughout a single orbit.

\section{Conclusion}

Based on calibration data and simulations, the use of p-terphenyl and SiPMs to develop a neutron spectrometer for a 3U CubeSat has been successful. INCA's launch later this year is expected to provide data that sheds a new light on the spectrum of albedo neutrons, furthering our understanding of the source of the proton population in the inner Van Allen belt. Following the proof of concept, similar compact neutron spectrometers could be flown on inner-heliospheric CubeSats, as piggy-backs on balloon missions to search for neutrons associated with terrestrial gamma-ray flashes, or as a planetary/solar probe. Data from a neutron spectrometer sent close to the Sun would be highly valuable, as it would give a brand new look at the spectrum of lower energy solar neutrons that decay before reaching $1 \mathrm{AU}$ [3].

\section{References}

[1] T.W. Armstrong, K. C. Chandler, J. Barish, Calculations of neutron flux spectra induced in the earth's atmosphere by galactic cosmic rays, Journal of Geophysical Research 78, no. 16 (1973): 2715-2726.

[2] G. Dietze, H. Klein, Gamma-calibration of NE 213 scintillation counters, Nuclear Instruments and Methods in Physics Research 193, no. 3 (1982): 549-556.

[3] G.A. de Nolfo et al. In ICRC Proceedings of Science (2019)

[4] C. Dyer, Radiation effects on spacecraft \& aircraft, In Solspa 2001, Proceedings of the Second Solar Cycle and Space Weather Euroconference, vol. 477, pp. 505-512. 2002.

[5] V. Jentsch, On the role of external and internal source in generating energy and pitch angle distributions of inner-zone protons, Journal of Geophysical Research: Space Physics 86, no. A2 (1981): 701-710.

[6] J.A. Lockwood, L. Hsieh, L. Friling, C. Chen, D. Swartz, Atmospheric neutron and gamma ray fluxes and energy spectra, Journal of Geophysical Research: Space Physics 84, no. A4 (1979): 1402-1408.

[7] M. Merker, Energetic neutrons leaking from the top of the atmosphere, Physical Review Letters 29, no. 22 (1972): 1531.

[8] A.M. Preszler, S. Moon, R. S. White, Atmospheric neutrons, Journal of Geophysical Research 81, no. 25 (1976): 4715-4722.

[9] A. Sardet, C. Varignon, B. Laurent, T. Granier, A. Oberstedt. p-Terphenyl: An alternative to liquid scintillators for neutron detection, Nuclear Instruments and Methods in Physics Research 792 (2015): 74-80.

[10] R.S. Selesnick, M. D. Looper, R. A. Mewaldt, A theoretical model of the inner proton radiation belt Space weather 5, no. 4 (2007).

[11] R.S. Selesnick, High-energy radiation belt electrons from CRAND, Journal of Geophysical Research: Space Physics 120, no. 4 (2015): 2912-2917.

[12] S.F. Singer, Trapped albedo theory of the radiation belt, Physical Review Letters 1, no. 5 (1958): 181.

[13] A.B. Smith, P. R. Fields, and J. H. Roberts, Spontaneous fission neutron spectrum of Cf 252 Physical Review 108, no. 2 (1957): 411.

[14] A. Zoglauer, R. Andritschke, S.E. Boggs, F. Schopper, G. Weidenspointner, C.B. Wunderer, MEGAlib: simulation and data analysis for low-to-medium-energy gamma-ray telescopes, In Space Telescopes and Instrumentation 2008: Ultraviolet to Gamma Ray, vol. 7011, p. 70113F. 